\title{
HIGH CONDUCTANCE IN RANDOM SUPERLATTICES WITH CORRELATED DISORDER
}

\author{
E. DIEZ', A. SÁNCHEZ ${ }^{1}$ and F. DOMÍNGUEZ-ADAME ${ }^{2}$ \\ 'Departamento de Matemáticas, Escuela Politécnica Superior, Universidad Carlos III, E-28911 Leganés, \\ Madrid, Spain \\ ${ }^{2}$ Departamento de Física de Materiales, Facultad de Fisicas, Universidad Complutense, E-28040 Madrid, \\ Spain
}

\begin{abstract}
We study d.c. conductance of disordered $\mathrm{GaAs}-\mathrm{Ga}_{1-x} \mathrm{Al}_{x} \mathrm{As}$ superlattices where the disorder is intentional and short-range correlated. We consider $\mathrm{Ga}_{1-} \mathrm{Al}_{x}$ As layers of the same thickness, where GaAs layers present two different thicknesses randomly distributed along the growth direction, with the constraint that one of them always appears in pairs. A set of almost unscattered electron states is observed in spite of the disorder, revealing itself through a distinct conductance maximum. Interestingly, this maximum is also detected in imperfect superlattices, where interface roughness is taken into account by allowing quantum-well thicknesses to fuctuate around their nominal values. Our predictions can be used to demonstrate experimentally that structural correlations inhibit the localization effects of disorder, even in the presence of imperfections.
\end{abstract}

Electron localization is inhibited and bands of extended states appear in one-dimensional random systems whenever disorder exhibits spatial correlations[1-8]. Unfortunately, there is no experimental evidence concerning their relevance on transport properties and their physical implications on the design of new electronic devices. Recent advances of molecular beam epitaxy (MBE), which allows for an excellent control of structural parameters of semiconductor superlattices (SLs), led us to suggest that these systems are ideal candidates to carry out experiments on delocalization[6,7]. Theoretical analyses of actual SLs should include fluctuations due to interface imperfections during growth processing. Since delocalization by spatial correlations is due to resonant tunneling effects[9], it is most important to elucidate whether inelastic scattering from unintentional interface roughness will strongly affect the quantum coherence required to observe delocalization. The aim of this work is to demonstrate on firm grounds that intentionally correlated disordered GaAs$\mathrm{Ga}_{1-x} \mathrm{Al}_{x}$ As SLs show a significative enhancement of the d.c. conductance in comparison with the uncorrelated ones, even if unintentionally the deviations of several monolayers from the wanted layer thickness occurs during growth.

We will focus on electron states close to the bottom of the conduction-band with $k_{\perp}=0$ and neglect the nonparabolicity effects hereafter. Then, a one-band effective-mass Hamiltonian suffices to obtain accurate results. For simplicity we assume the same effective-mass $m$ * at the $\Gamma$ valley in both semiconductors. We take the same barrier thickness $b$, whereas the thickness of each quantum-well ( $Q W$ ) is $\Delta z_{n}-b \equiv z_{n}-z_{n-1}-b, z_{n}$ being the position of the centre of the $n$th barrier in the growth direction. In the following we will consider that $\Delta z_{n}-b$ takes on only two values - $a$ and $a^{\prime}$ - in perfect SLs. A random dimer QW SL (DQWSL) is constructed by imposing the additional constraint that QWs of thickness $a^{\prime}$ appear only in pairs, called hereafter a dimer $Q W$ (DQW). Physical magnitudes of interest can be computed using a transfer-matrix formalism [7]. In particular, we can obtain the transmission coefficient $\tau(E, N)$ for an electron with energy $E$ in a SL with $N$ barriers, i.e. the dimensionless d.c. conductance at zero temperature[10].

$$
\kappa(E, N)=\frac{\tau(E, N)}{1-\tau(E, N)},
$$

and at finite temperature[11]

$$
\kappa(\mu, T)=\frac{\int\left(-\frac{\partial f}{\partial E}\right) \tau(E, N) \mathrm{d} E}{\int\left(-\frac{\partial f}{\partial E}\right)[1-\tau(E, N)]} \mathrm{d} E
$$

where integrations are extended over the allowed bands, $f$ is the Fermi-Dirac distribution and $\mu$ denotes the chemical potential of the sample.

As already mentioned, we are interested in the effects of interface roughness appearing during growth: protrusions of one semiconductor into the other cause in-plane disorder. It is well known that the average size of these defects depends on the growth conditions and, therefore, one is forced to develop a simple approach that holds valid for a large 
variety of samples. We describe the local excess or defect of monolayers by allowing $\Delta z_{n}$ to fluctuate uniformly around the nominal values $a+b$ or $a^{\prime}+b$. This approach enables us to restore in-plane translational symmetry. Our approximation should be valid whenever the mean-free-path of electrons is much smaller than the lateral average size of protrusions, as the electrons move in micro-QWs with a small area and uniform thickness. To get an accurate description of electron dynamics, an average over all possible configurations of disorder is needed because the number of interface defects, as well as their mean thickness, vary from layer to layer. For definiteness we take $\Delta z_{n}=a\left(1+W \varepsilon_{n}\right)+b$ or $\Delta z_{n}=a^{\prime}(1+W$ $\left.\varepsilon_{n}\right)+b$, where $W$ is a positive parameter measuring the maximum fluctuation and $\varepsilon_{n}$ is distributed according to a uniform probability distribution $P\left(\varepsilon_{n}\right)=1$ if $\left|\varepsilon_{n}\right|<1 / 2$ and zero otherwise. Note that $\Delta z_{n}$ is a random uncorrelated variable, even when its mean value follows the dimer constraint.

Let us consider electron transmission through a single DQW in an otherwise perfect and periodic SL. It is a matter of simple algebra to demonstrate that complete transparency $(\tau=1)$ takes place whenever the energy of the incident electron equals the resonant value $E_{\mathrm{r}}$ given by[7]

$$
\begin{array}{r}
\cos \left(\rho a^{\prime}\right) \cosh (\eta b)-\left(\frac{\rho^{2}-\eta^{2}}{2 \rho \eta}\right) \\
\sin \left(\rho a^{\prime}\right) \sinh (\eta b)=0,
\end{array}
$$

where $\rho^{2}=2 m^{*} E_{\mathrm{r}} / \hbar^{2}$ and $\eta^{2}=2 m^{*}\left(\Delta E_{\mathrm{c}}-E_{\mathrm{r}}\right) / \hbar^{2}$, $\Delta E_{\mathrm{c}}$ being the conduction-band offset, provided that the electron moves in an allowed miniband of the periodic SL. Notice that the position of the resonant energy is fixed from the values of the layer thicknesses and can be tailored by choosing appropriate parameters. Whenever the electron matches such an energy value, it remains unscattered by the DQW. Two important facts should be mentioned at this point. On the one hand, there exists a finite range of values of $a^{\prime}$ placing $E_{\mathrm{r}}$ within one allowed miniband in a given SL. Hence it is possible to locate $E_{\mathrm{r}}$ as close as we wish to the Fermi level. On the other hand, perfect transparency is not met whenever only one isolated $\mathrm{QW}$ of thickness $a^{\prime}$ is considered. Therefore, it is clear that a perfect transmission takes place due to resonant coupling between quasi-levels in the two QWs forming the DQW.

The above result does not imply anything about the existence of extended states in a SL with a finite number DQW. It is necessary to study that problem separately. Therefore we have evaluated the d.c. conductance at zero temperature, $\kappa(E, N)$, and at finite temperature, $\kappa(\mu, T)$ for both perfect and imperfect SLs close to the resonant energy $E_{\mathrm{r}}$. For comparison we have also considered perfect SLs with intentional disorder, but without the constraint of pairing. As typical values, we have taken $\Delta E_{\mathrm{c}}=250 \mathrm{meV}, m^{*}=0.067 \mathrm{~m}, m$ being the electron mass, $a=b=32 \AA$ and $a^{\prime}=26 \AA$. With these parameters there is only one miniband below the barrier in the periodic SL, ranging from 102 up to $175 \mathrm{meV}$, where energy is measured from the bottom of the conduction-band in GaAs. The resonant energy is found to be $E_{\mathrm{r}}=156 \mathrm{meV}$ from eqn (2) and thus it lies within this allowed miniband. The maximum number of barriers we have considered is $N=500$ and the number of QWs with thickness $a^{\prime}$ is $N / 5$. The d.c. conductance was averaged over 300 realizations, for $W$ ranging from 0 up to a maximum of 0.25 . This value amounts to having maximum fluctuations of $10 \AA$, well above the actual limits of MBE.

An example of the behaviour of the average d.c. conductance at $T=0 \mathrm{~K}$ around the resonant energy is shown in Fig. 1. The curves for single realizations behave in the same way, and the only effect of averaging is to smooth out particular features of realizations, keeping the main common characteristic, i.e. the distinct maximum close to the resonant energy $E_{\mathrm{r}}=156 \mathrm{meV}$. Curve (a) corresponds to perfect $(W=0)$ DWQSLs, whereas curves (b) and (c) show the effects of imperfections. Note that the dramatic increase of conductance around $E_{\mathrm{r}}$ remains even if $W>0$, although, as it should be expected, its value at $E_{\mathrm{r}}$ decreases on increasing $W$. However, this reduction of the conductance is not very significant if one considers that fluctuations can amount up to $3 \AA$ in Fig. $1(W=0.10)$. This is the reason why we claim that delocalization by correlated disorder is a robust phenomenon. Interestingly, this maximum is not observed when DQW are absent, even if unintentional fluctuations are neglected, as is plotted in curve (d).

The dependence of the conductance with the system size characterizes the spatial extent of the electronic states. In Fig. 2 we present results for electron

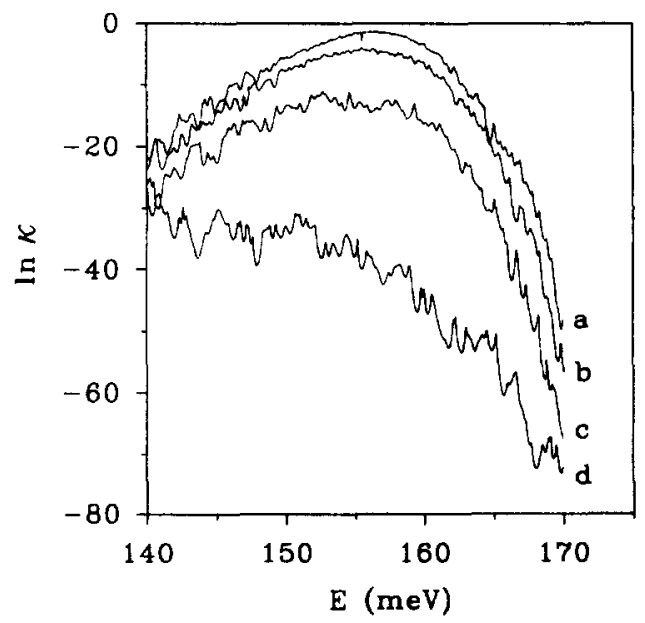

Fig. 1. Average d.c. conductance at zero temperature as a function of the electron energy for SLs with $N=500$ bartiers, $a=b=32 \AA$ and $a^{\prime}=26 \AA$, where resonant energy is $E_{\mathrm{r}}=156 \mathrm{meV}$. Curves correspond to SLs with DQWs and (a) $W=0$. (b) 0.05 , (c) 0.10 and (d) without DWQs and $\boldsymbol{W}=\mathbf{0}$. 


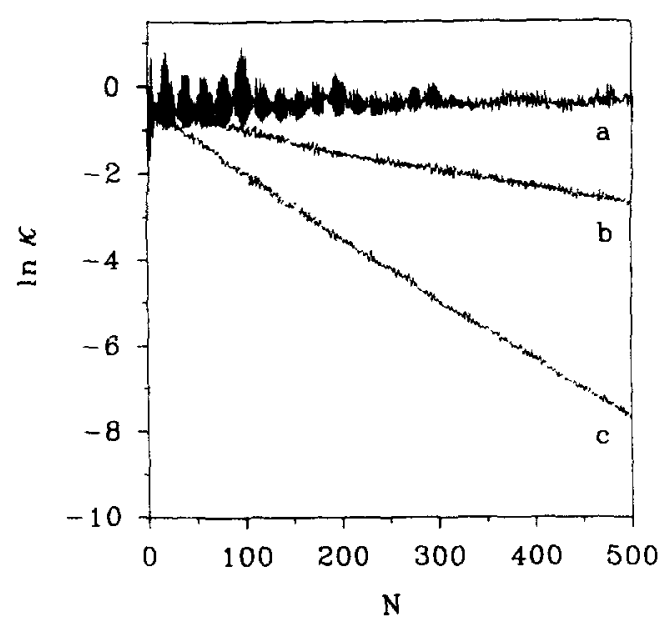

Fig. 2. Average d.c. conductance at zero temperature as a function of the number of barriers at the resonant energy $E_{\mathrm{r}}=156 \mathrm{meV}$ for random SLs with DWQs and (a) $\boldsymbol{W}=0$, (b) 0.05 and (c) 0.10. Parameters are the same as in Fig. I.

energy $E_{\mathrm{r}}$ in DQWSLs for the three different values of the parameter $W$, as in Fig. 1 , at $\mathrm{T}=0 \mathrm{~K}$. In perfect SLs $(W=0)$ the conductance presents a flat dependence on the system size, hence indicating a perfectly ohmic behaviour, and only small fluctuations around the mean value are detected. On the contrary, when imperfections are introduced $(W>0)$ we observe a nonohmic behaviour of the conductance decreasing exponentially with the system size. The dependence of the conductance with the number or barriers is of the form In $k \propto-\gamma(W) N$, where $\gamma(W)$ is the exponential decay rate. Figure 3 displays the dependence of this parameter on $W$ for random SLs with and without DQWs. In the absence of pairing, the least square fit gives $\gamma(W) \sim 0.076+W(W-0.015)$. Therefore, the behaviour is intrinsically nonohmic, even in the limit

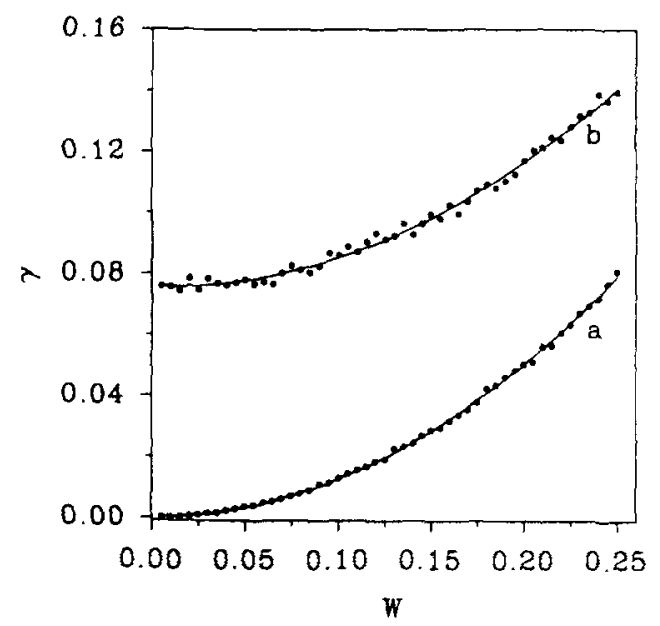

Fig. 3. Exponential decay rate $\gamma$ at the resonant energy $E_{\mathrm{r}}=156 \mathrm{meV}$ as a function of the fluctuation parameter $W$, for random SLs (a) with DWQs and (b) without DQW'. Solid lines represent least square fits. Parameters are the same as in Fig. 1.

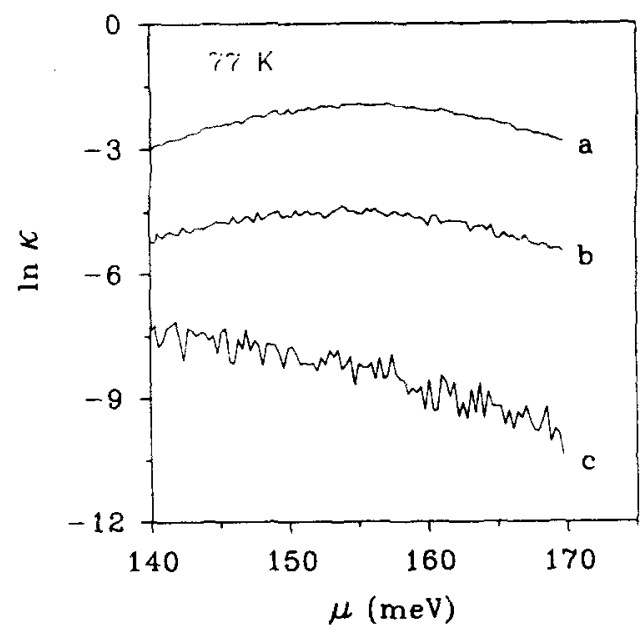

Fig. 4. Average d.c. conductance at $77 \mathrm{~K}$ as a function of the chemical potential for SLs with $N=500$ barriers, $a=b=32 \AA$ and $a^{\prime}=26 \AA$. Curves correspond to SLs with DQWs and (a) $W=0$, (b) 0.10 and (c) without DWQs and $W=0$.

$W \rightarrow 0$. On the contrary, in random DQWSLs this dependence is of the form $\gamma(W) \sim 1.28 W^{2}$. Such dependence indicates that for small values of $W$, which is currently available by means of MBE, the behaviour is almost ohmic.

It is also most important for experimental purposes to investigate whether finite-temperature effects will affect or not the predicted unscattered states and, particularly, their transport properties. To this end, we have computed the d.c. conductance by means of eqn $(\mathrm{lb})$ for the three kinds of SL that we are studying, namely DQWSL, DQWSL with fluctuations, and random SL without DQWs. In Fig. 4, the d.c. conductance at $77 \mathrm{~K}$ as a function of the chemical potential of the sample is seen for the three different SLs. A marked enhancement in the d.c. conductance pattern is clearly observed whenever the chemical potential lies close to $E_{\mathrm{r}}=156 \mathrm{meV}$ in perfect DQWSL [Fig. 4(a)]. This peak persists when we add imperfections during growth, even when the fluctuations are as large as $10 \%$ [Fig. 4(b)], and their only appreciable effect is a reduction of its height. On the contrary, this peak is not observed when DQWs are absent and the SL is purely random [Fig. 4(c)]. It is not difficult to understand why this is so. The derivative of the Fermi-Dirac function in eqn (1b) is very peaked around the chemical potential. Therefore, when the chemical potential lies close to the set of extended states, that is to say, close to the resonance, there will be positive contributions to the conductance due to conducting states, as shown in Fig. 4(a,b). Clearly, if there are no extended states as in ordinary random QWSLs, there will never exist positive contributions and the sample will always present small and featureless d.c. conductance, as it is seen in Fig. 4(c). 
To summarize, we have studied d.c. conductance in intentionally disordered $\mathrm{GaAs}-\mathrm{Ga}_{1_{-x}} \mathrm{Al}_{x}$ As SLs with and without DQWs. In periodic SLs with a single DQW, there exists a resonant energy for which the electronic states remain unscattered due to the resonant coupling between the neighbouring $\mathrm{QW}$ forming the dimer. One of the main points we have found is that these resonance effects also arise when a finite number of DQW are randomly placed in the $\mathrm{SL}$, in spite of the inherent disorder. These effects manifest themselves through an enhancement of the d.c. conductance whenever the electron energy (at $T=0$ ) or the chemical potential (at $T>0$ ) matches the resonant energy. Our present results strongly support the idea that a dramatic enhancement of the d.c. conductance should be experimentally observable in actual SLs with correlated disorder, provided that we set SL parameters in such a way that $E_{\mathrm{r}}$ lies close to the chemical potential. On the contrary, all these effects are completely absent in random SLs without the constraint of pairing. We feel that, however, the most significant point we have demonstrated is that imperfections inadvertently introduced during growth, which we have simulated by random variations of the nominal thicknesses of the QWs, do not have very significant effects on the delocalization of electronic states by correlated disorder, at least within the available MBE capabilities. This is indeed an interesting result from a practical point of view, since it means that deviations of several monolayers from the ideal values of the well thicknesses do not destroy the quantum coherence required to observe delocalization. It is important to stress that the study of delocalization goes beyond the mere conceptual interest and, actually, new devices may be developed based on this effect. For instance, it is possible to disregard all electron states other than unscattered ones and this may be of interest to design electronical filters.

Acknowledgement-It is with great pleasure that we thank collaboration and illuminating conversations with F. Agulló-Rueda. This work was supported by CICyT (Spain) under project MAT 95-0325.

\section{REFERENCES}

1. J. C. Flores, J. Phys. Condens. Matter 1, 8471 (1989)

2. P. Phillips and H.-L. Wu, Science 252, 1805 (1991).

3. P. K. Datta, D. Giri and K. Kundu, Phys. Rev. B 47, 10.727 (1993).

4. A. Sánchez and F. Dominguez-Adame, J. Phys. A 27, 3725 (1994).

5. A. Sánchez, E. Maciá and F. Dominguez-Adame, Phys. Rev. B 49, 147 (1994).

6. E. Diez, A. Sánchez and F. Dominguez-Adame, Phys. Rev. B 50, 14,359 (1994).

7. F. Dominguez-Adame, A. Sánchez and E. Diez, Phys. Rev. B 50, 17,736 (1994).

8. M. Hilke, J. Phys. A 27, 4773 (1994).

9. A. Sánchez, F. Dominguez-Adame, G. Berman and F. Izrailev, Phys. Rev. B 51, 6769 (1995).

10. R. Landauer, IBM J. Res. Dev. 1, 223 (1957).

11. H. L. Engquist and P. W. Anderson, Phys. Rev. B 24, 1151 (1981). 